\title{
Perimenopausal oestrogen could prevent breast cancer - For
}

\author{
Isaac Manyonda ${ }^{1}$, Vikram Talaulikar ${ }^{2}$, Roxanna Pirhadi ${ }^{3}$, John Ward ${ }^{1}$, Dibyesh Banerjee ${ }^{1}$, \\ and Joseph Onwude ${ }^{1}$ \\ ${ }^{1}$ Affiliation not available \\ ${ }^{2}$ University College London Hospitals NHS Foundation Trust \\ ${ }^{3}$ Anglia Ruskin University Faculty of Health Education Medicine \& Social Care
}

November 19, 2020

BJOG Debate

[Title] Perimenopausal oestrogen could prevent breast cancer - For

[Authors' title] Oestrogen replacement therapy and breast cancer

Isaac Manyonda, ${ }^{1}$ Vikram Talaulikar, ${ }^{2}$ Roxanna Pirhadi, ${ }^{3}$ John Ward, ${ }^{4}$ Dibyesh Banerjee, ${ }^{5}$ Joseph Onwude ${ }^{6}$

${ }^{1}$ Department of Obstetrics and Gynecology, St George's University Hospitals NHS Foundation Trust / St George's, University of London, London, United Kingdom.

2 Reproductive Medicine Unit, EGA Wing, University College London Hospital, 235 Euston Road, London NW1 2BU.

3 Faculty of Health, Education, Medicine and Social Care, Anglia Ruskin University, Second Floor William Harvey Building, Bishop Hall Lane, Chelmsford CM1 1SQ

${ }^{4}$ St George's, University of London, London, United Kingdom.

${ }^{5}$ Department of Surgery, St George's University Hospitals NHS Foundation Trust, London, United Kingdom.

${ }^{6}$ The Chelmsford Private Day Surgery Hospital, Fenton House, 85-89 New London Road, Chelmsford CM2 $0 \mathrm{PP}$.

Contemporary best clinical practice should be evidence-based, with the best evidence coming from systematic reviews / meta-analyses and randomised clinical trials (RCTs). The Women's Health Initiative (WHI) studies of hormone replacement therapy (HRT) provide robust evidence in their recent 20-year follow-up report that supports our supposition, that oestrogen is protective against breast cancer (Chlebowski et al. JAMA 2020;324(4):369-80). They have, at several time-points, previously reported consistently that whilst combined HRT (cHRT; oestrogen and progesterone) was associated with an increased incidence of breast cancer), oestrogen-only HRT (ERT: given to women with prior hysterectomies) was associated with a significantly reduced risk of developing or dying from the disease. The direct interpretation of the findings from these RCTs is that exposure to exogenous oestrogen (ERT) prevents breast cancer.

In opposition to the WHI findings are a number of observational studies, notably the "Collaborative Reanalysis" (Collaborative Group on Hormonal Factors in Breast Cancer. Lancet 1997;350:1047-59) and the "Million Women" (Million Women Study Collaborators. Lancet 2003;362:419-27) studies, which have reported increased risks of breast cancer from both cHRT and ERT. However, Shapiro et al (Shapiro et al. J Fam Plann Reprod Health Care 2012;38(2):102-9) critiqued both studies, examining factors including time order, bias, confounding, statistical stability, strength of association, dose-duration response, internal and external 
consistency and biologic plausibility, and concluded that the causality link reached by both studies was defective, and that both had low scientific validity because of serious significant epidemiological faults.

The group continues to publish along the same lines, and in October 2020 they reported an increased risk of breast cancer from a nested case-control study (Vinogradova et al. BMJ 2020;371:m3873). However, such a study design, which starts from disease (breast cancer) could never allow an estimation of the risk of developing breast cancer, but merely the risk of exposure to HRT. This study could be criticised on many other fronts, as already reflected in the rapid responses seen in the publishing journal. These studies cause untold alarm among women taking HRT, and fly in the face of advice from National Institute for Health and Care Excellence which says HRT should be offered to most women as the potential benefits far outweigh the risks.

What is also often notable in these reports of increased risk is the failure to report in context. The absolute risk from HRT is small: in 1000 women aged 50-60, who are otherwise healthy and not taking HRT, 23 will develop breast cancer over a five-year period \sout; \soutif a similar number take cHRT an additional 4 (27) will develop the disease, whilst by contrast there will be 4 less (19/1000) in women on ERT. To put these figures into perspective, a BMI of $>40$ will add 24 women; 2 units of alcohol per day will add 5 and smoking will add 3 , while 2 hours of exercise per week will reduce the risk by 7 women. In the context of lifestyle, the alarmist nature of reporting surrounding HRT and breast cancer becomes apparent.

Evidence from RCTs show a significant reduction in both incidence of and mortality from breast cancer in women who took ERT: this message should be presented clearly and robustly so that women benefit from the cheap, safe and effective hormone called oestrogen.

Disclosure of interest: All authors (IM, VST, RP, JW, DB, JO) report no conflict of interest. Completed disclosure of interest forms are available to view online as supporting information. 